\title{
Long noncoding RNA CASC7 inhibits the proliferation and migration of papillary thyroid cancer cells by inhibiting miR-34a-5p
}

Wencong Sun ${ }^{1,2,3}$ and Detao Yin ${ }^{1,3^{*}}$

\begin{abstract}
Long noncoding RNAs (IncRNAs) play an essential role in the progression of papillary thyroid cancer (PTC). However, the expression and function of IncRNA cancer susceptibility candidate 7 (CASC7) in PTC remain unknown. The purpose of this study was to investigate the role and molecular mechanism of CASC7 in regulating PTC cell behavior. The expression of CASC7, miR-34a-5p, and tumor protein P73 (TP73) was determined by qRT-PCR and western blot. Cell proliferation was examined by MTT assay. Cell apoptosis was assessed by flow cytometry following Annexin V and PI staining. Cell migration was determined by Transwell migration assay. The interaction between miR-34a-5p and CASC7 or TP73 was examined by luciferase reporter assay. CASC7 and TP73 expression were significantly lower, whereas miR-34a-5p expression was higher in PTC tissues than the adjacent normal tissues. Furthermore, CASC7 overexpression inhibited cell proliferation and migration, whereas facilitated cell apoptosis in human PTC cell lines (K1 and TPC-1). Mechanistically, CASC7 acted as a sponge of miR-34a-5p to upregulate TP73 expression. Moreover, miR-34a-5p mimic transfection could abate the CASC7-regulated PTC cell proliferation, migration, and apoptosis. Collectively, CASC7 inhibited the proliferation and migration of PTC cells by sponging miR-34a-5p to upregulate TP73 expression.
\end{abstract}

Keywords: CASC7, miR-34a-5p, TP73, Thyroid cancer

\section{Background}

Thyroid cancer (TC) is the most common endocrine malignancy, accounting for about $1 \%$ of all human malignant neoplasms $[1,2]$. TC can be classified into papillary thyroid cancer (PTC), follicular carcinoma, undifferentiated carcinoma, and medullary carcinoma according to the specific histological features [3]. PTC is the most prevalent form of thyroid malignancy, accounting for more than $80 \%$ of TC [4]. PTC has a relatively good prognosis, but there are still cases $(<10 \%)$ that present with tumors exhibiting aggressive characteristics [5]. Therefore, in-depth exploration of the mechanism of

\footnotetext{
*Correspondence: detaoyin@sohu.com

1 Department of Thyroid Surgery, The First Affiliated Hospital

of Zhengzhou University, No.1 Jianshe Road, Zhengzhou 450052, Henan, People's Republic of China

Full list of author information is available at the end of the article
}

PTC occurrence and development has significant clinical implications for the diagnosis and treatment of PTC.

Long noncoding RNAs (lncRNAs) are a class of RNA molecules with a length of more than 200 nucleotides that participate in various physiological or pathological processes [6]. LncRNAs have been a hotspot in cancer research. Considerable studies have confirmed that the abnormal expression of lncRNAs is closely related to the initiation and development of various cancers, including PTC $[7,8]$. Certain lncRNAs are lowly expressed in PTC tissues and have tumor-suppressive function in PTC, for example, H19 [9], maternally expressed 3 [10], and BRAF-activated non-protein coding RNA [11]. In contrast, certain lncRNAs that are highly expressed in PTC tissues to exert oncogenic function in PTC, for example, copy number amplified long noncoding RNA in papillary thyroid cancer 1 [12], IncRNA-activated by TGF-beta [13], and nuclear paraspeckle assembly transcript 1 [14]. 
The above-mentioned findings indicate that lncRNAs are important for understanding the molecular biology of PTC progression.

LncRNA CASC7 (cancer susceptibility candidate 7) is a $\sim 9.3 \mathrm{~kb}$ lncRNA with tumor-suppressive roles in some cancers. For instance, Zhang et al. demonstrated that CASC7 is lowly expressed in colorectal cancer tissues and suppresses colorectal cancer cell proliferation and migration via inhibiting microRNA (miR)-21 [15]. More recently, Gong et al. reported that CASC7 is downregulated in glioma tissues and inhibits the progression of glioma via regulating $\mathrm{Wnt} / \beta$-catenin signaling pathway [16]. However, the expression and function of CASC7 in other cancers remain largely undefined, and the present study was designed to explore its role and molecular mechanism in PTC.

\section{Materials and methods Human sample collection}

Thirty PTC tissues and adjacent normal tissues were obtained from patients who underwent surgical treatment for PTC at our hospital. Samples were stored at $-80^{\circ} \mathrm{C}$ until use. The diagnosis of PTC was confirmed histopathologically by an experienced pathologist. The study was approved by the Ethics committee of First Affiliated Hospital of Zhengzhou University, and all participants have signed a written informed consent for using the specimens.

\section{Cell culture}

Human PTC cell lines (K1 and TPC-1) were purchased from BeNa Culture Collection (Beijing, China). Cells were maintained in high-glucose Dulbecco's modified Eagle's medium (DMEM; Gibco, Thermo Fisher Scientific, Inc., Waltham, MA, USA) supplemented with $10 \%$ fetal bovine serum (FBS; Gibco), glutamine, and sodium pyruvate in an incubator at $37{ }^{\circ} \mathrm{C}$ with $5 \% \mathrm{CO}_{2}$.

\section{Cell transfection}

To overexpress CASC7, the full-length gene sequence of CASC7 was synthesized and subcloned into a pcDNA3.1 vector (Invitrogen, Thermo Fisher Scientific, Inc.), generating pcDNA3.1-CASC7. The empty pcDNA3.1 vector was used as the control. The short hairpin RNAs (shRNAs) against CASC7 (sh-CASC7), scrambled oligonucleotides (sh-NC), miR-34a-5p mimic, mimic negative control (NC), miR-34a-5p inhibitor, and inhibitor NC were purchased from GenePharma (Shanghai, China). For transfection, K1 and TPC-1 cells in the logarithmic growth phase were seeded in 6-well cell culture plates at a density of $5 \times 10^{3} / \mathrm{mL}$ and incubated overnight at $37{ }^{\circ} \mathrm{C}$. When the cells reached a confluency of $70 \sim 80 \%$, cells were transfected with these vectors and/or miRNA mimic/inhibitor using Lipofectamine 2000 (Invitrogen) according to the manufacturer's instructions. Following $48 \mathrm{~h}$ of transfection, cells were harvested and processed for further analysis.

\section{Cell viability and apoptosis assay}

For cell viability assay, cells were plated in 96-well cell culture plates at a density of $3 \times 10^{5}$ cells $/ \mathrm{mL}$ with $100 \mu \mathrm{L}$ culture medium. After incubation for $24 \mathrm{~h}, 20 \mu \mathrm{L}$ of MTT $(5 \mathrm{mg} / \mathrm{mL})$ was added to each well and the cells were incubated for an additional $4 \mathrm{~h}$. Subsequently, the culture medium was removed and $150 \mu \mathrm{L}$ of DMSO was added to each well. The absorbance in each well was measured at $490 \mathrm{~nm}$ using a microplate reader.

Cell apoptosis was measured by flow cytometry using the Annexin V-FITC/PI Apoptosis Kit (KeyGENE Biotechnology, Nanjing, China) according to the manufacturer's instructions. The resulting fluorescence was measured by flow cytometry using the Becton Dickinson FACSCalibur (Becton Dickinson, Franklin Lakes, NJ, USA).

\section{Transwell migration assay}

After different transfection treatments, K1 and TPC-1 cells were resuspended in $100 \mu \mathrm{L}$ serum-free medium $\left(5 \times 10^{4}\right.$ cells $)$ was added into the upper chambers of Transwell inserts $(8.0-\mu \mathrm{m}$ pore size) for migration (without Matrigel) assays. The lower chambers were filled with $500 \mu \mathrm{L}$ DMEM medium containing 10\% FBS. After $24 \mathrm{~h}$, the bottom surface of the membrane was fixed in $10 \%$ formaldehyde solution, followed by $1 \%$ crystal violet staining for $2 \mathrm{~h}$. The number of migrated cells was calculated and cells were imaged under a microscope from five random fields.

\section{Quantitative real-time PCR (qRT-PCR)}

Total RNA was extracted from tissues or cell lines using RNAisoPlus Kit (Takara, Dalian, China). cDNA synthesis was performed with $1 \sim 2 \mu \mathrm{g}$ of RNA using iScript First Strand cDNA synthesis kit (Bio-Rad, Hercules, CA, USA). After reverse transcription, the expression of CASC7 and tumor protein P73 (TP73) were detected using the SYBR premix (Takara) whereas the expression of miR-34a-5p was using the miRNA qRT-PCR kit (GeneCopoeia, Rockville, MD, USA) in Applied Biosystems 7500 PCR system (Applied Biosystems, Foster, CA, USA). U6 was employed as the endogenous control for miR-34a-5p and GAPDH for CASC7 and TP73. The primers were synthesized by Shanghai Bioengineering Co. (Shanghai, China). 


\section{Western blot}

Total protein was extracted from the cells in RIPA lysis buffer (Beyotime, Shanghai, China). Then equal protein $(20 \mu \mathrm{g})$ from cell lysates was separated by $10 \%$ SDS-PAGE gels and electro-transferred onto PVDF membranes (Millipore, Billerica, MA, USA). The membranes were then incubated with the primary antibodies against TP73 (1:1000; Abcam, Cambridge, MA, USA) and GAPDH (1:2500; Abcam) overnight at $4{ }^{\circ} \mathrm{C}$, followed by the horseradish peroxidase (HRP)-conjugated secondary antibodies (1:2000; Santa Cruz Biotechnology, Dallas, TX, USA). Detection was performed using an enhanced chemiluminescence (ECL) detection kit (Millipore).

\section{Luciferase reporter assay}

The recombination luciferase plasmids containing the wildtype (WT) or mutant (Mut) gene sequences of CASC7 or TP73 3 '-UTR region with miR-34a-5p binding site were constructed from Genecopoeia company (Rockville, Md, USA). K1 cells were cotransfected with the luciferase construct along with miR-34a-5p mimic and mimic NC. After $48 \mathrm{~h}$ of transfection, the luciferase activity was examined using the luciferase assay kit (Promega, Madison, WI, USA) according to the manufacturer's instructions.

\section{Statistical analysis}

Statistical analysis was performed using SPSS version 20.0 (IBM, Chicago, IL, USA). The unpaired Student's $t$ test and one-way analysis of variance were used to analyze differences between two or more groups, respectively. $P<0.05$ was considered to have a statistically significant difference.

\section{Results}

\section{CASC7 overexpression inhibited PTC cell proliferation} and migration, and induced PTC cell apoptosis

qRT-PCR analysis showed that CASC7 expression was significantly lower in most PTC tissues than that in adjacent normal tissues (Fig. 1a). Furthermore, CASC7 expression showed a downward trend with the PTC stage progression (Additional file 1: Figure S1). To examine the functional role of CASC7 in regulating PTC cell behavior, we overexpressed CASC7 in two human PTC cell lines (K1 and TPC-1). The overexpression efficiency of CASC7 was confirmed by qRT-PCR analysis in both cells (Fig. 1b). CASC7 overexpression led to impaired cell growth (Fig. 1c) and enhanced cell apoptosis (Fig. 1d). Furthermore, Transwell migration assay demonstrated that the number of migrated cells in PTC cells following CASC7 overexpression was notably less than that in those transfected with empty vector (Fig. 1e).

\section{CASC7 negatively regulated miR-34a-5p expression, whereas positively regulated TP73 expression}

miR-34a-5p expression was notably higher, whereas TP73 mRNA expression was lower in most PTC tissues when compared with the adjacent normal tissues (Fig. 2a). Furthermore, CASC7 expression was inversely correlated with miR-34a-5p expression, whereas positively correlated with TP73 mRNA expression in PTC tissues (Fig. 2b). Then, we evaluated the effect of CASC7 overexpression on expression of miR-34a-5p and TP73. qRT-PCR and western blot analyses showed that CASC7 overexpression noticeably downregulated miR-34a-5p expression in both $\mathrm{K} 1$ and TPC-1 cells (Fig. 2c, f). Conversely, CASC7 overexpression resulted in a significant upregulation of TP73 expression, both at mRNA (Fig. 2d, g) and protein (Fig. 2e, h) levels. On the contrary, CASC7 silencing notably decreased mRNA and protein levels of TP73 in both cells (Additional file 2: Figure S2A-D).

\section{CASC7 acted as a sponge of miR-34a-5p to upregulate TP73 expression}

The results of bioinformatics analysis using the prediction tools miRanda (http://www.microrna.org/) showed that CASC7 harbors putative binding sites of miR-34a-5p, which was predicted to target the $3^{\prime}$-UTR of TP73 using TargetScan (http://www.targetscan.org/vert_72/) (Fig. 3a). Accordingly, we investigated the interaction between miR-34a-5p and CASC7 as well as miR-34a-5p and TP73. Luciferase reporter assay showed that miR-34a-5p mimic transfection significantly inhibited luciferase activity in the CASC7 WT but not CASC7 Mut group, indicating the direct interaction between CASC7 and miR-34a-5p. Furthermore, the miR-34a-5p mimic notably repressed luciferase activity in cells co-transfected with TP73-WT reporter, whereas had no obvious effect on the luciferase activity in TP73-Mut group, verifying TP73 was a direct target of miR-34a-5p (Fig. 3a).

Moreover, TP73 mRNA and protein levels were significantly downregulated in both $\mathrm{K} 1$ and TPC-1 cells following introduction of miR-34a-5p mimic. In contrast, inhibition of miR-34a-5p by miR-34a-5p inhibitor transfection

\footnotetext{
(See figure on next page.)

Fig. 1 CASC7 overexpression inhibited PTC cell proliferation and migration, and induced cell apoptosis. a qRT-PCR analysis of CASC7 expression in PTC tissues $(n=30)$ and adjacent normal tissues $(n=30)$. b CASC7 expression determined by qRT-PCR analysis, c cell viability assessed by MTT assay, d cell apoptosis determined by flow cytometry following Annexin V and PI staining, and e cell migration assessed by Transwell migration assay in human PTC cell lines (K1 and TPC-1) transfected with the CASC7 overexpression vector and empty vector. ${ }^{* *} P<0.01$ vs. Tumor-adjacent tissues or Vector. Data are expressed as the mean \pm standard deviation $(n=3)$.
} 


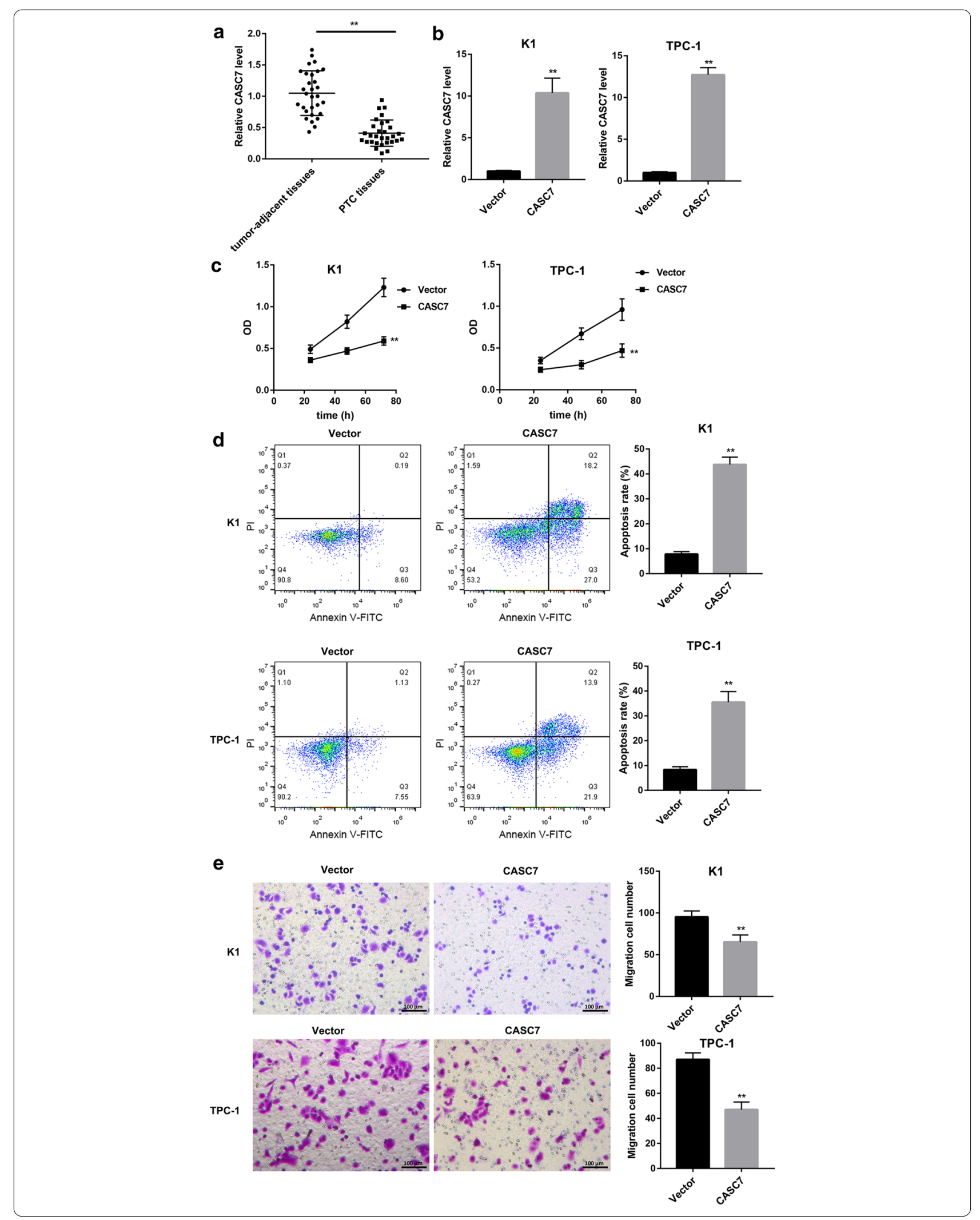




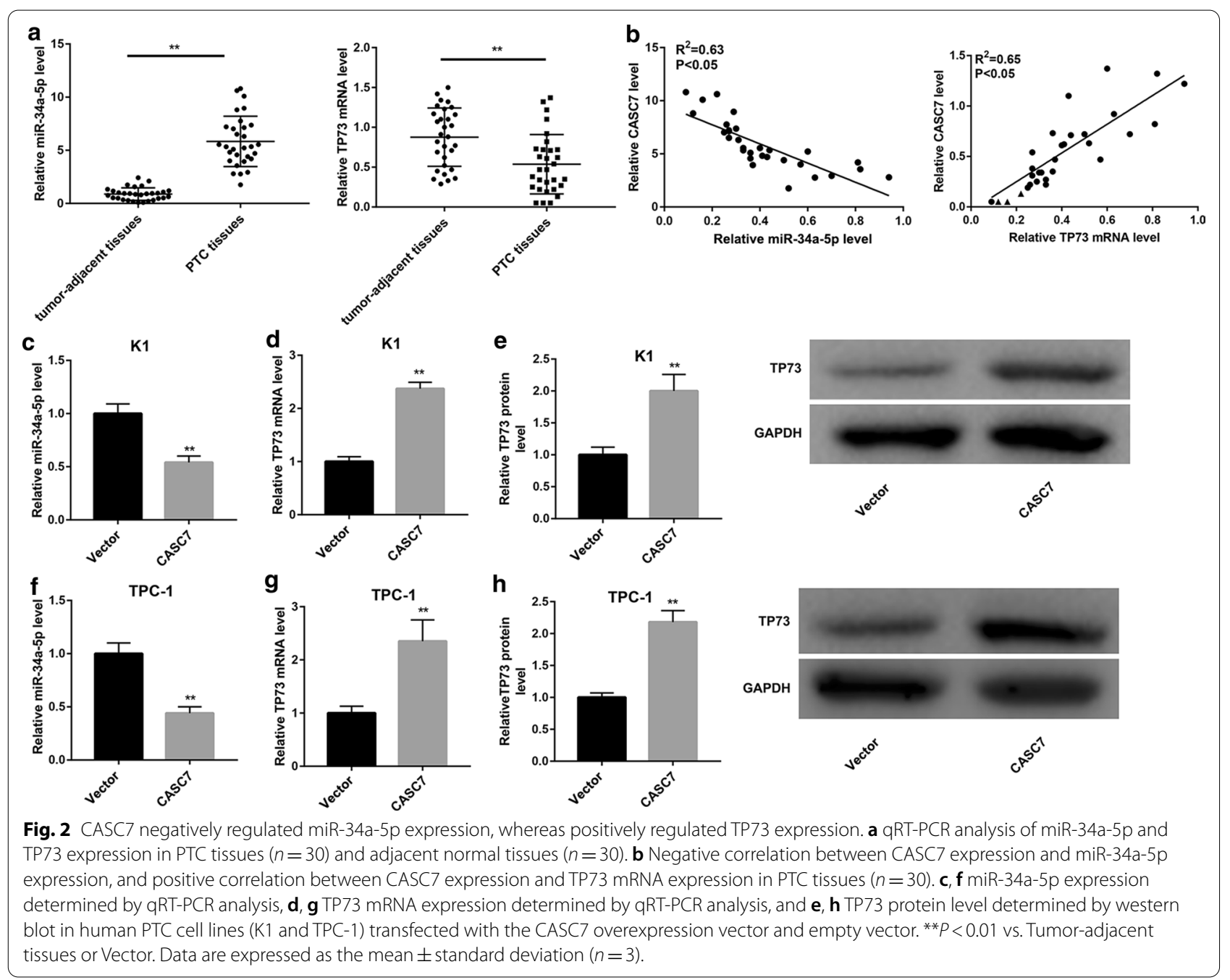

markedly upregulated TP73 mRNA and protein levels in both cells (Fig. 3b-e).

Importantly, CASC7 overexpression led to a notable upregulation of TP73 mRNA and protein levels in both cells. We also found that the miR-34a-5p mimic-mediated inhibition of TP73 expression could be abrogated by CASC7 overexpression (Fig. $4 \mathrm{a}-\mathrm{d}$ ). Taken together, these data indicated that CASC7 could function as a sponge of miR-34a-5p to increase TP73 expression.

\section{CASC7-regulated PTC cell behavior through sponging miR-34a-5p}

Finally, we determined whether CASC7 regulates PTC cell behavior through sponging miR-34a-5p. In contrast to CASC7 overexpression, miR-34a-5p mimic transfection significantly promoted cell proliferation (Fig. 5a) and migration (Fig. 5c), whereas inhibited cell apoptosis (Fig. 5b) in both $\mathrm{K} 1$ and TPC-1 cells. More importantly, the CASC7 overexpression-mediated inhibition of cell proliferation and migration and promotion of cell apoptosis could be abated following introduction of miR34a-5p mimic (Fig. 5a-c).

\section{Discussion}

In the present study, we investigated the expression of CASC7, miR-34a-5p and TP73 in PTC tissues. CASC7 and TP73 expressions were found to be significantly downregulated, whereas miR-34a-5p was upregulated in PTC tissues. Furthermore, we explored their interaction and role in regulating PTC cell behavior. Our results showed that CASC7 acted as a sponge of miR-34a-5p to upregulate the expression of tumor-suppressive TP73, and thereby inhibited PTC cell proliferation and migration and induced PTC cell apoptosis.

CASC7 has been shown to be involved in several diseases, such as asthma and myocardial ischemia-reperfusion injury $[17,18]$. With regard to cancer, CASC7 has been reported in glioma and colorectal cancer where 


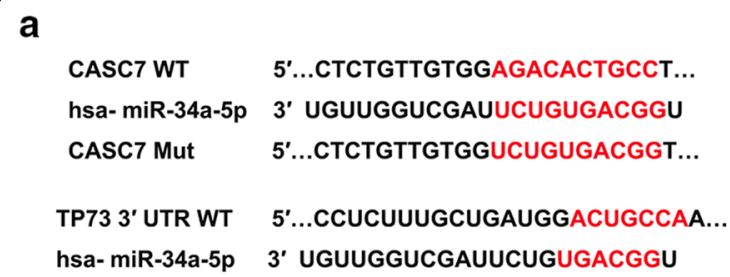

b

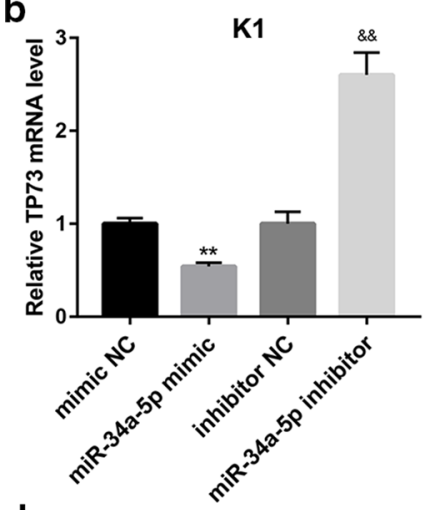

d

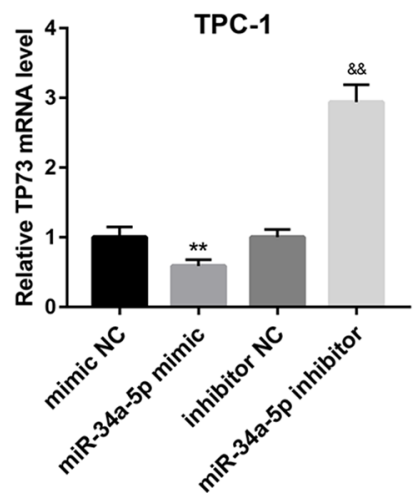

C

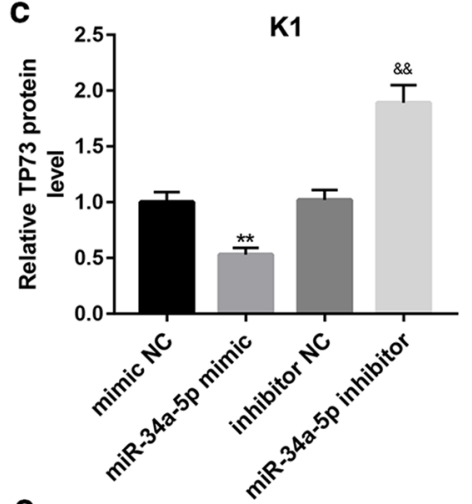

e

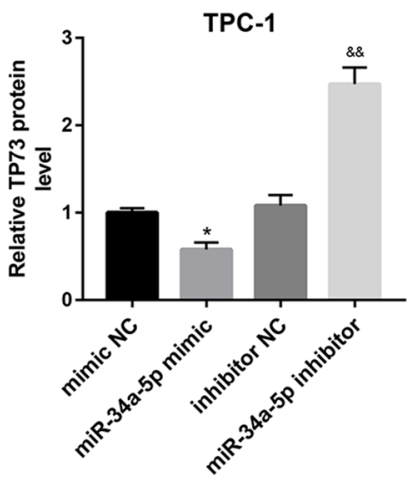

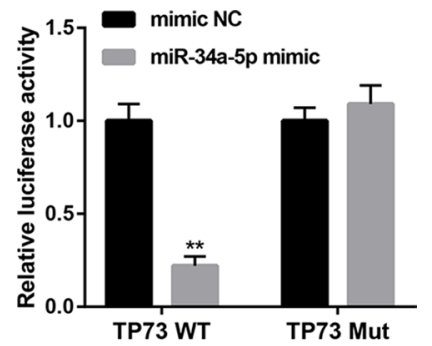

TP73

GAPDH

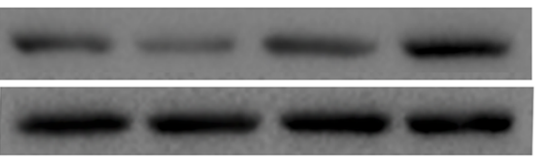

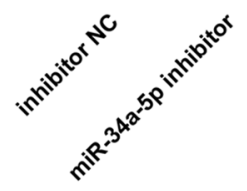
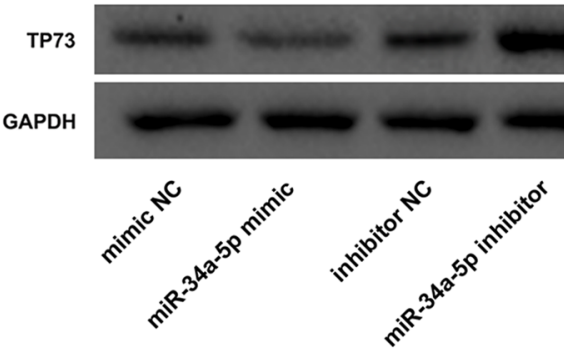

Fig. 3 TP73 was a target of miR-34a-5p. a Prediction of target relationship between miR-34a-5p and CASC7 or TP73. Luciferase reporter assay was performed to evaluate the interaction ability between miR-34a-5 $p$ and CASC7 or TP73 3'-UTR. b, d TP73 mRNA expression determined by qRT-PCR analysis, and $\mathbf{c}$, e TP73 protein level determined by western blot in human PTC cell lines (K1 and TPC-1) co-transfected with miR-34a-5p mimic/mimic NC and miR-34a-5p inhibitor/inhibitor NC. ${ }^{*} P<0.05$, ${ }^{*} P<0.01$ vs. mimic NC; ${ }^{\& \&} P<0.01$ vs. inhibitor NC. Data are expressed as the mean \pm standard deviation $(n=3)$.

CASC7 exerted tumor-suppressive roles $[15,16]$. However, the expression and function of CASC7 in other cancers remains far from being elaborated. To our knowledge, this study is the first to show the downregulated expression and tumor-suppressive role of $\mathrm{CASC} 7$ in PTC. Our results also revelaed that CASC7 expression showed a downward trend with PTC stage progression. However, further investigation was required to elucidate the association of CASC7 expression with clinicopathological features of PTC.

MiRNAs are small noncoding RNA molecules $(21 \sim$ 23 nucleotides) that play an important role in regulating various cellular processes such as cell proliferation, differentiation, and apoptosis [19]. A study by Wang et al. revealed that miR-34a-5p expression is significantly higher in PTC plasma samples and tissues relative to healthy controls [20], indicating that miR-34a-5p might play a crucil role in PTC progression. MiR-34a-5p exerts tumor-suppressive or oncogenic role in cancer [21]. For example, miR-34a-5p overexpression has been demonstrated to inhibit ovarian cancer cell proliferation and trigger apoptosis [22]. In contrast, Jing et al. reported that miR-34a-5p plays a tumor-promoting role in clear cell renal cell carcinoma [23]. Consistent with the oncogenic 


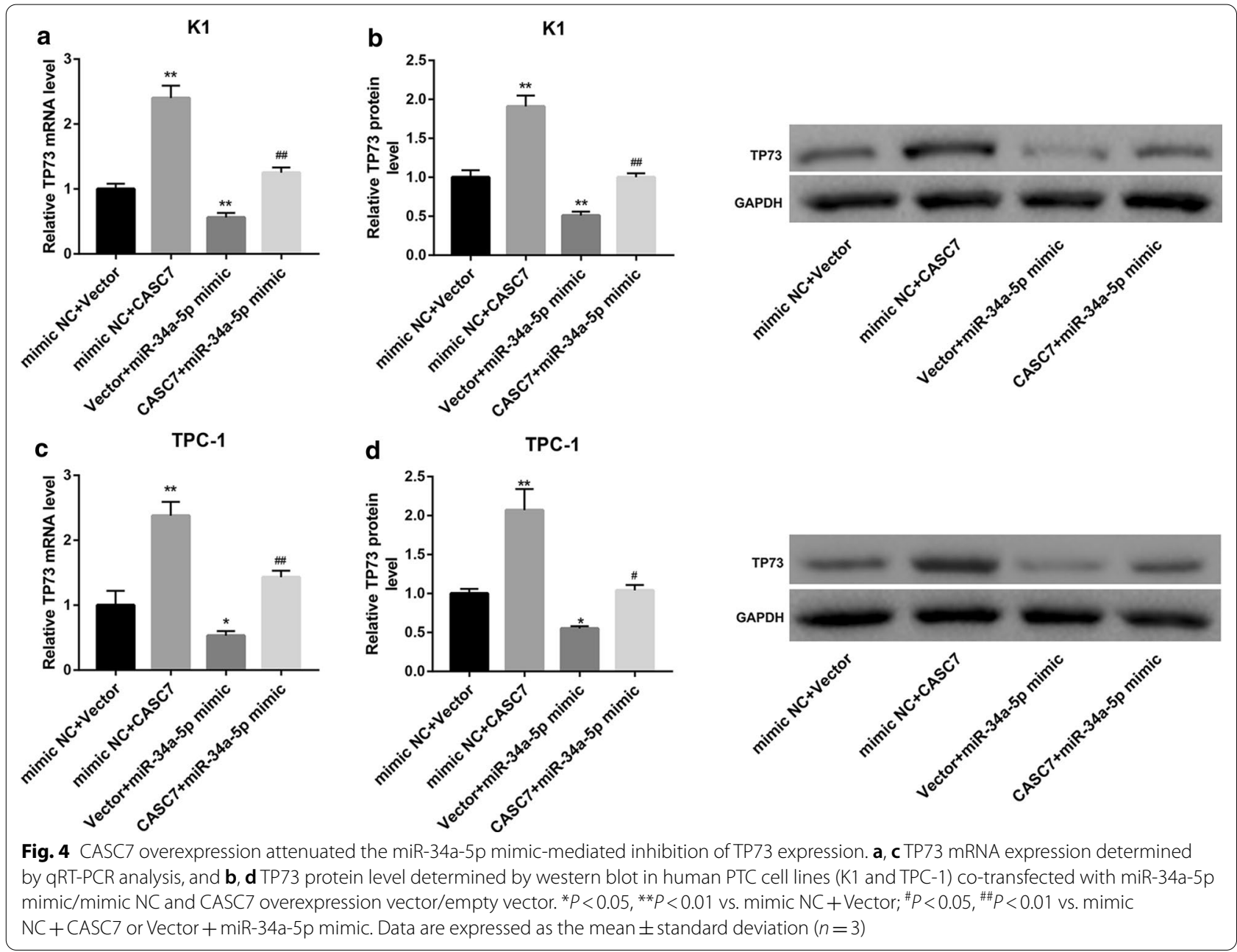

role, our results showed that miR-34a-5p mimic promoted cell proliferation and migration, and inhibited cell apoptosis in both $\mathrm{K} 1$ and TPC-1 cells. To our knowledge, this study is the first to demonstrate the oncogenic role of miR-34a-5p in PTC cells.

It has been well accepted that lncRNAs can function as competing endogenous RNAs (ceRNAs) or as molecular sponges in modulating the expression and biological functions of miRNAs in various cellular context $[24,25]$. Increasing lncRNAs have been reported to participate in the progression of PTC through the ceRNA mechanism $[14,26]$. For instance, lncRNA TUG1 (taurine upregulated gene 1) contributed to the progression of PTC cells through regulating miR-145/zinc finger E-box binding homeobox 1 (ZEB1) pathway [27]. Similarly, CASC7 can also regulate cancer development through acting as a ceRNA [15]. The TP73 gene, located on chromosome 1p36.3, encodes a product that shares significant structural homology with the tumor suppressor TP53 [28]. Abundant studies have characterized TP73 as a tumor-suppressive gene [28-30]. Using bioinformatics analysis and luciferase reporter assay, we confirmed TP73 as a direct target of miR-34a-5p. Furthermore, CASC7 can bind to miR-34a-5p and inhibit miR-34a-5p expression in PTC cells. Importantly, the miR-34a-5p-mediated targeted suppression of TP73 and promotion of PTC cell growth were abrogated when CASC7 was overexpressed in PTC cells. Thus, we conclude that CASC7 acts as a ceRNA of miR-34a-5p, leading to the derepression of the miR-34a-5p target TP73, and eventually regulated PTC cell behavior.

\section{Conclusion}

In summary, the findings in the present study demonstrate that CASC7 functions as a ceRNA of miR-34a-5p by sponging miR-34a-5p to upregulate its target TP73, thereby inhibiting PTC cell proliferation and migration and inducing PTC cell apoptosis. Our study extends the knowledge on the mechanism underlying PTC progression and 


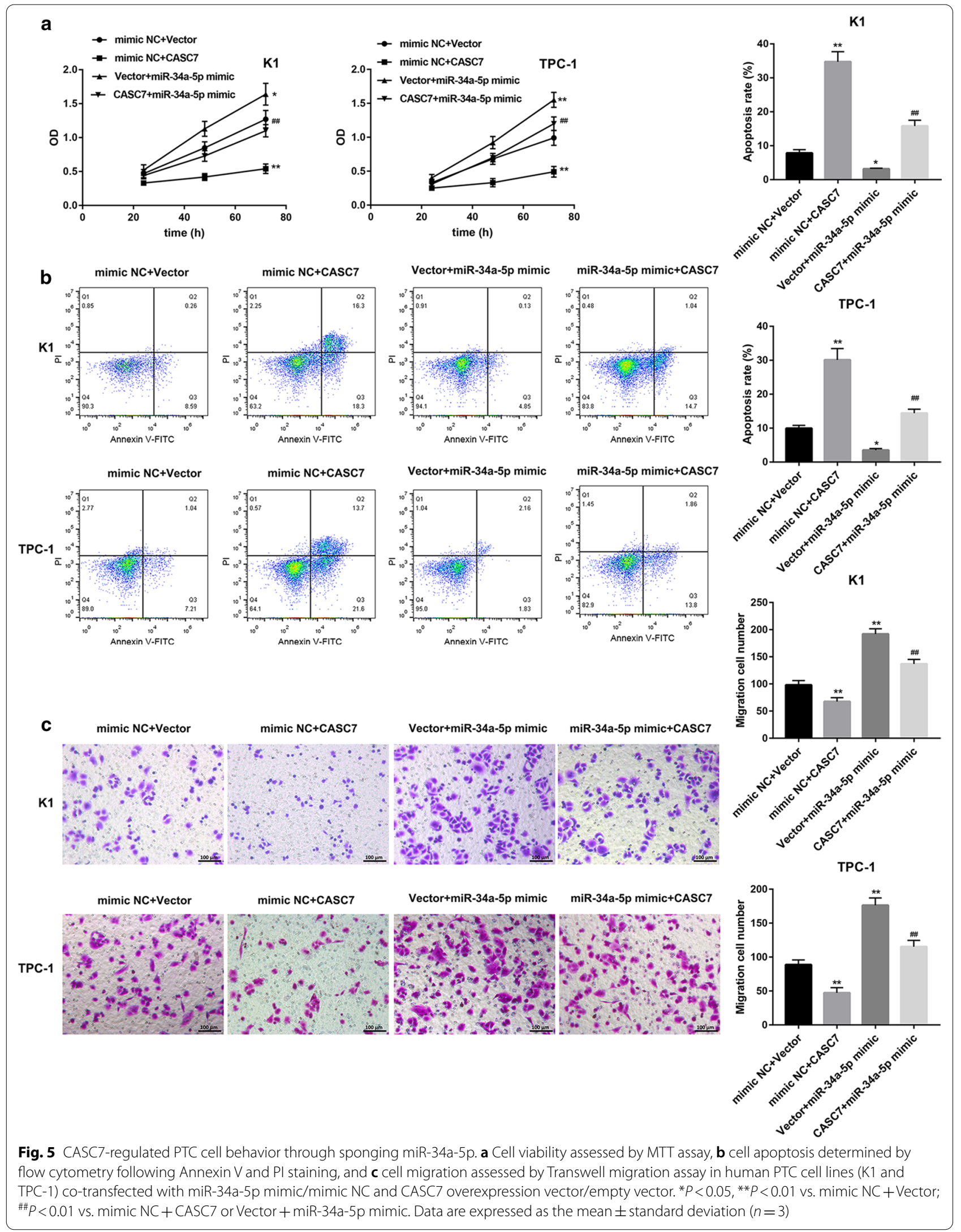


provides a new perspective for CASC7-directed drug targets for PTC.

\author{
Abbreviations \\ TC: Thyroid cancer; PTC: Papillary thyroid cancer; IncRNA: Long noncod- \\ ing RNA; CASC7: Cancer susceptibility candidate 7; miR: MicroRNA; DMEM: \\ Dulbecco's modified Eagle's medium; FBS: Fetal bovine serum; NC: Negative \\ control; shRNA: Short hairpin RNA; qRT-PCR: Quantitative real-time PCR; TP73: \\ Tumor protein P73; WT: Wild type; Mut: Mutant; ceRNA: Competing endog- \\ enous RNA; TUG1: Taurine upregulated gene 1.
}

\section{Supplementary Information}

The online version contains supplementary material available at https://doi. org/10.1186/s12576-021-00793-2.

Additional file 1: Figure S1. qRT-PCR analysis of CASC7 expression in PTC tissues (PTC-I $n=14$; PTC- $-1 \mid n=4$; PTC -|| $\mid n=9$; PTC-IV n=3) and adjacent normal tissues $(n=30){ }^{* * *} P<0.01$ vs. Tumor-adjacent tissues.

Additional file 2: Figure S2. (A, C) TP73 mRNA expression determined by qRT-PCR analysis, and (B, D) TP73 protein level determined by western blot in human PTC cell lines (K1 and TPC-1) transfected with the sh-CASC7 or sh-NC. ${ }^{* *} P<0.01$ vs. sh-NC. Data are expressed as the mean \pm standard deviation $(n=3)$.

\section{Acknowledgements}

Not applicable.

\section{Authors' contributions}

WS designed the study; WS and DY performed the experiments; WS and DY analyzed the data; WS drafted and revised the manuscript. All authors read and approved the final manuscript.

\section{Funding}

This study was supported by funding from the National Natural Science Foundation of China (81372863); Foundation of Henan university of science and technology innovation team funding (19IRTSTH002).

\section{Availability of data and material}

The datasets used in the current study are available from the corresponding author on reasonable request.

\section{Ethics approval and consent to participate}

The study was approved by the Ethics committee of First Affiliated Hospital of Zhengzhou University.

\section{Consent for publication}

All participants have signed a written informed consent for using the specimens.

\section{Competing interests}

The authors declare no conflict of interest.

\begin{abstract}
Author details
${ }^{1}$ Department of Thyroid Surgery, The First Affiliated Hospital of Zhengzhou University, No.1 Jianshe Road, Zhengzhou 450052, Henan, People's Republic of China. ${ }^{2}$ Department of Thyroid Surgery, Henan Provincial People's Hospital, People's Hospital of Zhengzhou University, No.7 Weiwu Road, Zhengzhou 450003, Henan, People's Republic of China. ${ }^{3}$ Key Discipline Laboratory of Clinical Medicine of Henan, Daxue Road, Zhengzhou 450050, Henan, People's Republic of China.
\end{abstract}

Received: 1 July 2020 Accepted: 11 February 2021

Published online: 11 March 2021

\section{References}

1. Davies L, Welch HG (2006) Increasing incidence of thyroid cancer in the United States, 1973-2002. JAMA 295(18):2164-2167

2. Boos LA, Schmitt A, Moch H, Komminoth P, Simillion C, Marinoni I, Nikiforov YE, Nikiforova MN, Perren A, Dettmer MS (2019) MiRNAs are involved in tall cell morphology in papillary thyroid carcinoma. Cancers (Basel) 11(6):885

3. Liu Z, Hu D, Huang Y, Chen S, Zeng W, Zhou L, Zhou W, Wang M, Feng H, Wei W et al (2019) Factors associated with distant metastasis in pediatric thyroid cancer: evaluation of the SEER database. Endocr Connect 8(2):78-85

4. Oda H, Miyauchi A, Ito Y, Yoshioka K, Nakayama A, Sasai H, Masuoka H, Yabuta T, Fukushima M, Higashiyama T et al (2016) Incidences of unfavorable events in the management of low-risk papillary microcarcinoma of the thyroid by active surveillance versus immediate surgery. Thyroid 26(1):150-155

5. Perisa MM, Sarcevic B, Troselj KG, Grsic K, Sitic S, Seiwerth S (2017) Expression of nm23-H1 and COX-2 in thyroid papillary carcinoma and microcarcinoma. Oncol Lett 13(5):3547-3555

6. Kwok ZH, Tay Y (2017) Long noncoding RNAs: lincs between human health and disease. Biochem Soc Trans 45(3):805

7. Schmitt AM, Chang HY (2017) Long noncoding RNAs: at the intersection of cancer and chromatin biology. Cold Spring Harbor Perspect Med. https ://doi.org/10.1101/cshperspect.a026492

8. Murugan AK, Munirajan AK, Alzahrani AS (2018) Long noncoding RNAs: emerging players in thyroid cancer pathogenesis. Endocr Relat Cancer 25(2):R59-r82

9. Lan X, Sun W, Dong W, Wang Z, Zhang T, He L, Zhang H (2018) Downregulation of long noncoding RNA H19 contributes to the proliferation and migration of papillary thyroid carcinoma. Gene 646:98-105

10. Wang C, Yan G, Zhang Y, Jia X, Bu P (2015) Long non-coding RNA MEG3 suppresses migration and invasion of thyroid carcinoma by targeting of Rac1. Neoplasma 62(4):541-549

11. Zhang J, Du Y, Zhang X, Li M, Li X (2018) Downregulation of BANCR promotes aggressiveness in papillary thyroid cancer via the MAPK and PI3K pathways. J Cancer 9(7):1318-1328

12. Chen C, Zhou L, Wang H, Chen J, Li W, Liu W, Shen M, Liu H, Fu X (2018) Long noncoding RNA CNALPTC1 promotes cell proliferation and migration of papillary thyroid cancer via sponging miR-30 family. American journal of cancer research 8(1):192-206

13. Fu XM, Guo W, Li N, Liu HZ, Liu J, Qiu SQ, Zhang Q, Wang LC, Li F, Li CL (2017) The expression and function of long noncoding RNA InCRNA-ATB in papillary thyroid cancer. Eur Rev Med Pharmacol Sci 21(14):3239-3246

14. Zhang H, Cai Y, Zheng L, Zhang Z, Lin X, Jiang N (2018) Long noncoding RNA NEAT1 regulate papillary thyroid cancer progression by modulating miR-129-5p/KLK7 expression. J Cell Physiol 233(10):6638-6648

15. Zhang Z, Fu C, Xu Q, Wei X (2017) Long non-coding RNA CASC7 inhibits the proliferation and migration of colon cancer cells via inhibiting microRNA-21. Biomed Pharmacother 95:1644-1653

16. Gong $X$, Liao $X$, Huang M (2019) LnCRNA CASC7 inhibits the progression of glioma via regulating Wnt/beta-catenin signaling pathway. Pathol Res Pract 215(3):564-570

17. Liao B, Gao F, Lin F, Yang S, Xu Z, Dong S (2019) LncRNA CASC7 inhibits myocardial apoptosis in myocardial ischemia-reperfusion rats by regulating MiR-21 expression. Panminerva Medica

18. Liu JH, Li C, Zhang CH, Zhang ZH (2020) LncRNA-CASC7 enhances corticosteroid sensitivity via inhibiting the PI3K/AKT signaling pathway by targeting miR-21 in severe asthma. Pulmonology 26(1):18-26

19. Bartel DP (2004) MicroRNAs: genomics, biogenesis, mechanism, and function. Cell 116(2):281-297

20. Wang Z, Lv J, Zou X, Huang Z, Zhang H, Liu Q, Jiang L, Zhou X, Zhu W (2019) A three plasma microRNA signature for papillary thyroid carcinoma diagnosis in Chinese patients. Gene 693:37-45

21. Li YY, Tao YW, Gao S, Li P, Zheng JM, Zhang SE, Liang J, Zhang Y (2018) Cancer-associated fibroblasts contribute to oral cancer cells proliferation and metastasis via exosome-mediated paracrine miR-34a-5p. EBioMedicine 36:209-220

22. Ding N, Wu H, Tao T, Peng E (2017) NEAT1 regulates cell proliferation and apoptosis of ovarian cancer by miR-34a-5p/BCL2. OncoTargets Ther 10:4905-4915 
23. Jing Z-F, Bi J-B, Li Z, Liu X, Li J, Zhu Y, Zhang X-T, Zhang Z, Li Z, Kong C-Z (2019) Inhibition of miR-34a-5p can rescue disruption of the p53-DAPK axis to suppress progression of clear cell renal cell carcinoma. Mol Oncol 13(10):2079-2097

24. Wang D, Xu H, Wu B, Jiang S, Pan H, Wang R, Chen J (2019) Long noncoding RNA MALAT1 sponges miR-124-3p.1/KLF5 to promote pulmonary vascular remodeling and cell cycle progression of pulmonary artery hypertension. Int J Mol Med 44(3):871-884

25. Zheng Y, Yu K, Huang C, Liu L, Zhao H, Huo M, Zhang J (2019) Integrated bioinformatics analysis reveals role of the LINC01093/miR-96-5p/ ZFAND5/NF-kB signaling axis in hepatocellular carcinoma. Exp Ther Med 18(5):3853-3860

26. Gao Y, Wang F, Zhang L, Kang M, Zhu L, Xu L, Liang W, Zhang W (2020) LINC00311 promotes cancer stem-like properties by targeting miR330-5p/TLR4 pathway in human papillary thyroid cancer. Cancer Med. https://doi.org/10.1002/cam4.2815

27. Lei H, Gao Y, Xu X (2017) LncRNA TUG1 influences papillary thyroid cancer cell proliferation, migration and EMT formation through targeting miR145. Acta Biochim Biophys Sin 49(7):588-597
28. Dong S, Pang JC, Hu J, Zhou LF, Ng HK (2002) Transcriptional inactivation of TP73 expression in oligodendroglial tumors. Int J Cancer 98(3):370-375

29. Ozaki T, Nakagawara A (2005) p73, a sophisticated p53 family member in the cancer world. Cancer Sci 96(11):729-737

30. Jiang $H$, Cheng L, Hu P, Liu R (2018) MicroRNA663b mediates TAM resistance in breast cancer by modulating TP73 expression. Mol Med Rep 18(1):1120-1126

\section{Publisher's Note}

Springer Nature remains neutral with regard to jurisdictional claims in published maps and institutional affiliations.
Ready to submit your research? Choose BMC and benefit from:

- fast, convenient online submission

- thorough peer review by experienced researchers in your field

- rapid publication on acceptance

- support for research data, including large and complex data types

- gold Open Access which fosters wider collaboration and increased citations

- maximum visibility for your research: over $100 \mathrm{M}$ website views per year

At BMC, research is always in progress.

Learn more biomedcentral.com/submissions 